\title{
NeuroRegulation
}

\section{When Discussing Neurofeedback, Does Modality Matter?}

\author{
Genomary Krigbaum, Psy.D..$^{1 *}$ and Nancy L. Wigton, Ph.D. ${ }^{1,2}$ \\ ${ }^{1}$ Grand Canyon University, College of Doctoral Studies, Phoenix, Arizona, USA \\ ${ }^{2}$ Applied Neurotherapy Center, LLC; Scottsdale, Arizona, USA
}

*Address correspondence to: Genomary Krigbaum, Psy.D., Grand Canyon University, College of Doctoral Studies, 3300 W Camelback Rd., Phoenix, Arizona, 85017. Email: genomary.krigbaum@my.gcu.edu

Copyright: (C 2014 Krigbaum and Wigton. This is an Open Access article distributed under the terms of the Creative Commons Attribution License (CC-BY).

\begin{abstract}
Over the years, several new models and variations of neurofeedback (NF) have been developed. As such, NF has grown from traditional amplitude based modalities to now include slow cortical potential NF, as well as various approaches grounded in QEEG technology, including $z$-score NF models. These differing modalities have important implications in terms of outcomes, the number of sessions required, and treatment specificity. This, in turn, impacts clinical practice, research, and marketing considerations. In an effort to gain some perspective for where the field is today, a comparative review is presented to illustrate the importance of noting what particular modality is being referenced when discussing NF.
\end{abstract}

Keywords: neurofeedback, traditional NF, slow cortical potential NF, QEEG-guided NF, zscore NF

\section{Introduction}

If evaluating neurofeedback (NF) from an evidence-based perspective is important, and we propose that it is, then distinguishing between modalities is an essential component of that process. Over the years, several new models and variations of NF have been developed, which directly impacts practice implications in terms of outcomes, the number of sessions required, and treatment specificity. Thus, noting which modality of NF is being implemented is a necessary step in discussing any NF intervention. 
In the 1970s, when NF was first being developed by the likes of Joe Kamiya, Barry Sterman, and Joel Lubar, the approach was generally the same, that of targeting amplitudes of specific frequency bands. However, since then, many other models, and variations of models, have been developed. While the standard for much of the history of NF was the use of only one or two electrode sites to train various EEG frequencies, today there are new models which use as many as 19 electrodes while incorporating real-time database metrics and targeting cortical regions of interest. Therefore, the current range of NF modalities include traditional amplitude-based NF (Theta-Beta, Sensory-Motor Rhythm [SMR], Alpha-Theta), slow cortical potential (SCP) NF, quantitative EEG (QEEG) guided NF (QNF), and z-score NF (ZNF) models including 4-channel, 19-channel surface, and low resolution brain electromagnetic tomography (LORETA) ZNF (also see Figure 1, which presents a graphic of this collection of models, in timeline format, to provide a better context for the development of these models over time).

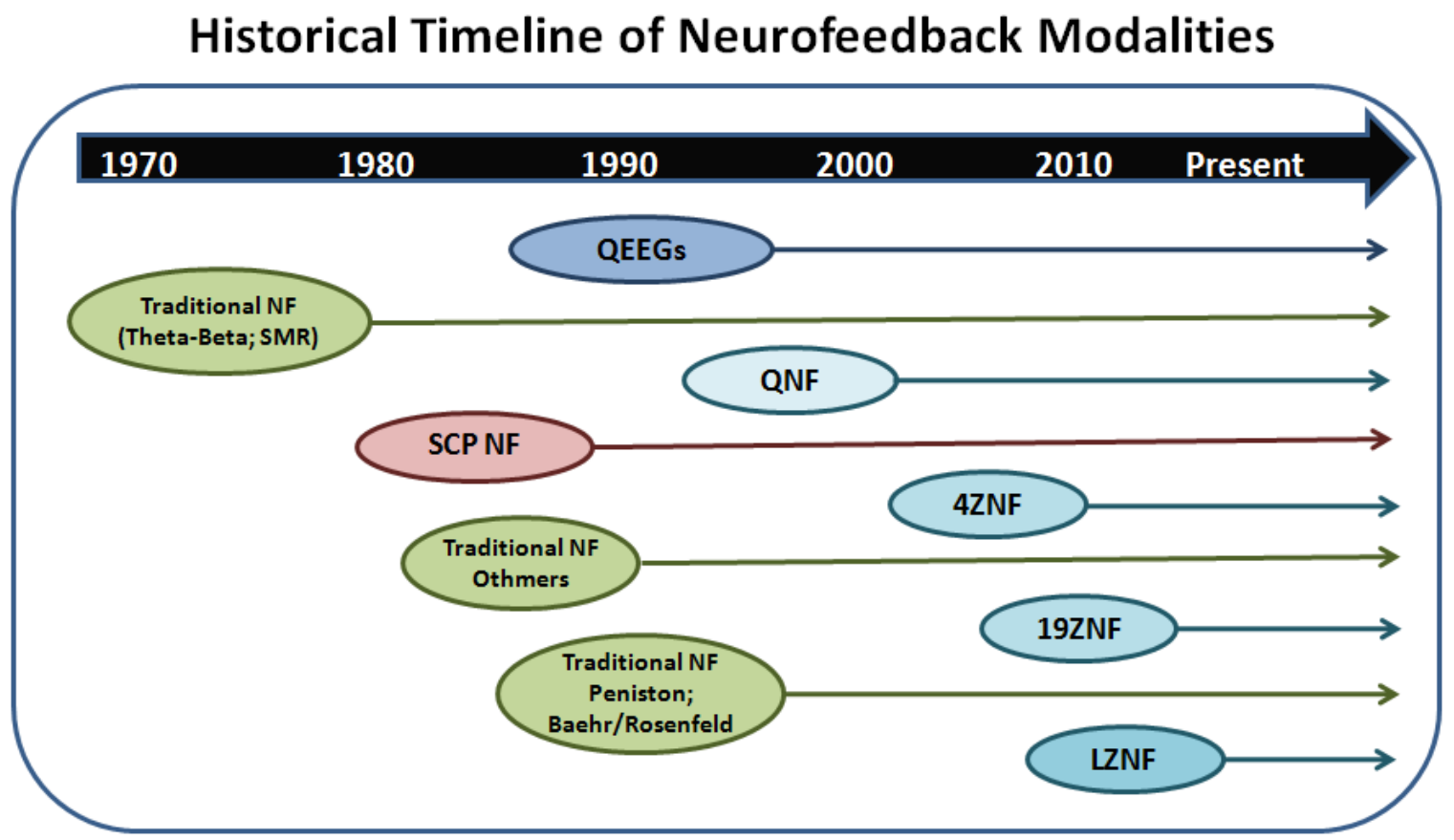

Figure 1. Timeline of when various neurofeedback modalities emerged.

NF models based on Theta-Beta bands and SCP have robust evidence-based research support; however, treatment sessions number from thirty to over forty and the specificity is limited to surface neuroregulation. In contrast, the evidence-based research is limited in newer models, such as the 19-channel and LORETA z-score models, which have been around since 2006 when the NF landscape changed to include ZNF. Yet, treatment outcome reports of the 19-channel and LORETA $z$-score models indicate that treatment goals can be achieved in fewer sessions, as more networks and deeper structures can be targeted (Thatcher, 2013; Wigton, 2013). Hence, depending on the modality, the amount of treatment sessions and specificity will make a difference in how NF is understood and/or is identified as a choice of treatment. For this reason, when addressing NF, the length of treatment and specificity are important in order to identify what modality of NF is being referenced. This is also important in terms of being an informed consumer of NF research in the literature (scientific). 
In 2012, the American Academy of Pediatrics (AAP) elevated biofeedback (to include NF) to a Level 1/Best Support recommended intervention. However, the three NF studies evaluated to make this determination (PracticeWise, 2012) only included Theta-Beta and SCP NF (Beauregard \& Levesque, 2006; Gevensleben et al., 2009; Levesque, Beauregard, \& Mensour, 2006), and did not include newer models (i.e., QNF or ZNF). As a result, it is important to be aware of the scope of this recommendation. Moreover, the non-inclusion of newer models (such as QNF or ZNF) may highlight the need for further empirical support, with solid methodologies, so as to elevate the evidence-based support matching that of Theta-Beta and SCP models; because the modality of NF used guides the treatment outcome, specificity and the number of sessions, which may vary between modalities.

Although all NF is informed by a basic framework of operant conditioning and learning theory (Sherlin et al., 2011), the variation in the application of that framework presents the NF clinician and researcher with many choices. Consequently, this comparative review is presented to gain some perspective of where the field is today with respect to the traditional NF, SCP NF, QNF, and ZNF models.

It is important to note there are other neuroregulation applications, which are also frequently referred to as NF, and are beyond the scope of this article. These include hemoencephalography (HEG), low-energy neurofeedback system (LENS), low intensity pulsed electromagnetic field ( $p E M F)$ stimulation, audio-visual entrainment (AVE), and real-time functional magnetic resonance imaging (fMRI) feedback, some of which may be considered adjuncts to NF treatment. So, due to the growing variety of neuroregulation techniques included under the overarching umbrella of NF, with differences in treatment outcome, number of sessions, and specificity, it is critical to be clear about which modality is being referred to when discussing NF.

Synopsis of NF Modalities, Treatment Outcomes, Number of Sessions, and Specificity.

Traditional NF. In the context of this review, the term traditional is used to describe the models that are primarily amplitude based. These are the longest standing iterations of NF and can be traced back to the original founders of the field (Kamiya, 1968; Sterman \& Friar, 1972; Lubar \& Shouse, 1976), including models where the targeted frequencies are ThetaBeta, SMR, or Alpha-Theta. While most NF practitioners who have been in the field for more than a decade are likely to be familiar with these models, newer entrants who may start with learning more recent NF models may not. Therefore, those not familiar with the historical aspects of NF are encouraged to acquaint themselves with the beginning works in the NF field (see Budzynski, 1999; Evans \& Abarbanel, 1999; Robbins, 2000; Thatcher \& Lubar, 2009). Other models, which have developed from this perspective, are symptom-based approaches such as the earlier Othmer models (Othmer, Othmer, \& Kaiser, 1999) where, generally, one frequency band is rewarded (either $12-15 \mathrm{~Hz}$ or $15-18 \mathrm{~Hz}$ ), while two other frequencies (Theta and High Beta) are inhibited. Still other models, which can be traced back to Kamiya's work, target parietal Alpha and/or Theta frequencies to enhance relaxation and creativity states (Budzynski, 1999), which Peniston and Kullkosky $(1990,1991)$ developed further, leading to treatment models for post-traumatic stress disorders and alcoholism. Finally, Baehr, Rosenfeld, and Baehr (1997) created protocols with the goal of balancing frontal alpha as a treatment for depression.

Slow Cortical Potential (SCP) NF. As described by Mayer, Wyckoff, and Strehl (2013), SCPs are very slow brain activation electrical shifts, generated subcortically and cortically, which alternate between being electrically negative or positive. Further, central to this model is an event related potential termed the contingent negative variation $(\mathrm{CNV})$, such that 
reduced CNVs have been found to be associated with attention deficit hyperactivity disorder (ADHD) symptoms; and SCP feedback has been shown to lead to an increased CNV.

Therefore, in SCP NF, with an active electrode at $\mathrm{Cz}$, the training goal is to generate shifts between surface-positive and surface-negative SCPs. The sessions typically consist of two to four runs made up of approximately 40 trials in each run. SCP NF is different from many traditional NF models in that there is only a single protocol used, with only slight variants, and has been designated by Mayer et al. as a one size fits all approach. Reports investigating the self-regulation of SCPs date back to the mid to late 1980s (Roberts, Rockstroh, Lutzenberger, Elbert, \& Birbaumer, 1989; Rockstroh, Birbaumer, Elbert, \& Lutzenberger, 1984; Rockstroh et al., 1993).

Traditional NF and SCP NF treatment outcomes, number of sessions, and specificity. Over time, the Theta-Beta and SMR variations of traditional NF, together with SCP NF, have come to be some of the most researched, specifically for the condition of ADHD (Arns, Heinrich, \& Strehl, 2014). This is further demonstrated by many reviews and meta-analyses available (Arns, de Ridder, Strehl, Breteler, \& Coenen, 2009; Arns et al., 2014; Brandeis, 2011; Gevensleben, Rothenberger, Moll, \& Heinrich, 2012; Lofthouse, Arnold, Hersch, Hurt, \& DeBeus, 2012; Mayer et al., 2013; Niv, 2013; Pigott, De Biase, Bodenhamer-Davis, \& Davis, 2013). Overall, this collection of literature provides strong support for NF being considered efficacious for ADHD.

While the traditional NF models may vary with respect to which EEG frequencies are trained, elements common to them are targeting amplitudes and a need for 40+ sessions, on average, for successful clinical outcomes. Regarding SCP, in their review of SCP NF studies for ADHD, Mayer et al. (2013) report the number of sessions ranged from 25 to 35 . Thus, while SCP NF is frequently investigated and reported alongside traditional NF, it is possible that SCP NF may require somewhat fewer sessions that traditional NF.

However, with a reliance on only one or two electrode placements, specificity in traditional and SCP NF is limited. At any individual electrode location, the EEG recording includes diffuse sources (both close and distant) that are picked up from other brain areas (Thatcher, 2013). Thus, with traditional NF, EEG recordings from an electrode at $\mathrm{Cz}$ will actually include a blend of the EEG signal from all other areas of the scalp, to varying degrees. Yet, traditional NF still employs choices of different frequencies at varying sites, depending on the particular symptoms or case presentation. However, with SCP NF, generally there is a single electrode on the central strip, typically at $\mathrm{Cz}$, with a singular protocol applied to all clients (Mayer et al., 2013).

QEEG-guided NF (QNF). In their recent review of NF for ADHD, Arns et al. (2014) suggest that research efforts should focus on NF protocols that are tailored to the individual, as many studies have suggested clinical outcomes can be improved with a personalized approach to NF. QNF allows for a tailored approach to individual treatment. QNF is grounded in QEEG technology dating back to the late 1980s; it has been improved through the years, so that the treatment can be tailored given the particular QEEG baseline, clinical status, and history of the client (Arns, Drinkenburg, \& Kenemans, 2012). This then allows for individualized treatments of differing electrocortical presentations, even with the same or similar overarching diagnoses (Hammond, 2010). Multiple reports in the literature support this clinical approach indicating how training the deviant $z$-scores towards the mean (i.e., normalize the QEEG) yields clinical benefit (Arns et al., 2012; Breteler, Arns, Peters, Giepmans, \& Verhoeven, 2010; Collura, 2008a; Orgim \& Kestad, 2013; Surmeli, Ertem, Eralp, \& Kos, 2012; Surmeli \& Ertem, 2009, 2010; Walker, 2009, 2010a, 2011, 2012a). Therefore, QNF still targets amplitudes of frequency bands, but does so in an effort to 
normalize the excessive $z$-score deviations shown in the QEEG that correspond to the overall clinical picture.

QNF treatment outcomes, number of sessions, and specificity. QNF studies are well represented in peer-reviewed literature; however, no meta-analysis of QNF has been identified to-date. Yet, the current QNF studies in the literature do report positive treatment outcomes. Moreover, while traditional and SCP NF mostly focus on ADHD symptoms, QNF studies report covering a wider range of symptoms, syndromes and disorders that include, behavior aspects, mood, cognitive dysfunction, epilepsy, head injuries, autism spectrum, migraines, learning disorders, schizophrenia, post-traumatic stress disorder, Down syndrome, and intellectual disability (Arns et al., 2012; Breteler et al., 2010; Coben \& Myers, 2010; Huang-Storms, Bodenhamer-Davis, Davis, \& Dunn, 2006; Koberda, Hillier, Jones, Moses, \& Koberda 2012; Surmeli et al., 2012; Surmeli \& Ertem, 2007, 2009, 2010, 2011; Walker, 2009, 2010b, 2011, 2012b, 2013). This literature collection largely represents retrospective investigations from clinical settings.

While some QNF studies provide results from research where the number of sessions was in the range of around 20 sessions (Breteler et al., 2010; Walker, 2009) others have reported as many as 50 or 100 sessions (Surmeli \& Ertem, 2009, 2011). However, as with traditional NF, the number of sessions for QNF typically remains on an average of 40 (Thatcher, 2013; Wigton, 2013).

Specificity with QNF, however, improves by being able to individually tailor the protocol to the needs of the client as directed by the QEEG findings and clinical assessment. This is accomplished with protocols that target specified sites with a goal of normalizing identified deviant $z$-scores. Thus, this NF modality takes into account the heterogeneity of QEEG patterns, more so leveraging the sensitivity, reliability, and specificity of the QEEG, as discussed by Hammond (2010).

Z-Score NF (ZNF). Rather than targeting frequency amplitudes, as with traditional NF or QNF, in ZNF, the training targets are the calculated real-time QEEG $z$-scores integrated into the NF software. As a result, real-time QEEG assessment metrics can be paired with operant conditioning and incorporated into the NF session (Collura, 2014; Thatcher, 2013). Thus, ZNF capitalizes on the statistical foundation for a normal distribution, where a value converted to a $z$-score is a measure of the distance from the mean of a population, such that the mean represents a range considered to be typical (Collura, 2014). This brings a new dynamic to the NF process. The focus is no longer on making more or less of a particular frequency, but rather moving excessive live (i.e., real-time) $z$-score metrics towards the mean (i.e., $z=0$ ), thereby placing more emphases on normalizing the QEEG values. ZNF allows for more metrics to be targeted in a NF protocol as active training components - up to ten frequency bands, both absolute and relative power, frequency power ratios, and the connectivity metrics of asymmetry, coherence, and phase lag. Yet, consistent with being an outgrowth of the QNF model, normalization goals are still governed by the presenting clinical picture. ZNF is one of the newest models, but even within this category, three distinctions can be made to include 4-channel ZNF, surface 19-channel ZNF, and LORETA ZNF; each are discussed as follows:

4-Channel ZNF (4ZNF). The 4ZNF model was the first iteration and was introduced in 2006, with the total number of metrics available to train being 248 (Collura, 2014). However, while the reference to 4-channel implies only four channels can be trained, there are also options for one or two channel ZNF training as well; thus this can be considered an up to 4 channel 
model. Here the $z$-scores are calculated from the surface recordings; yet, there is still access to all metrics/components as described above (i.e., power, connectivity, etc.).

19-Channel ZNF (19ZNF). 19ZNF was made available as an outgrowth from 4ZNF in 2009, and the total number of metrics for protocol selection is 5700 (Collura, Thatcher, Smith, Lambos, \& Stark, 2009). Again, in 19ZNF, the surface EEG recording is the source for the real-time $z$-score calculations. However, unique to 19ZNF, in addition to the linked-ears montage, is the availability to directly train the Laplacian montage. Yet, as with 4ZNF, so long as the linked-ears montage is applied, any number of channels up to 19 can be included in a protocol, thus allowing the use of any number of electrodes from one to 19.

LORETA ZNF (LZNF). While there have been investigations of non-z-score LORETA NF (examples include Cannon, Lubar, Sokhaze, \& Baldwin, 2008; Cannon, Congedo, Lubar, \& Hutchens, 2009; Cannon, Baldwin, Diloreto, Phillips, Shaw, \& Levy, 2014), LZNF was first introduced in 2010. Even though similar in many respects, there is a fundamental difference between surface ZNF and LZNF. Surface ZNF calculates the $z$-score of identified EEG metrics at various 10-20 electrode sites, whereas with LZNF, the $z$-score is calculated for a particular collection of current source density (CSD) voxels. This makes it possible to conduct NF with the $z$-scores of the calculated location of deeper cortical dipole generating regions or structures (i.e., Brodmann areas, cingulate gyrus, precuneus, etc.). Consequently, isolated cortical regions of interest can be identified for normalization with this modality.

ZNF treatment outcomes, number of sessions, and specificity. Currently, case reviews, technical reports, and discussion about ZNF can be found in books (Collura, 2014; Thatcher, 2012), and publications such as that of Collura (2008b), Genardi (2012), Koberda (2012), Thatcher $(2008,2013)$, and Wigton (2013). Additionally, there have been conference presentations such as Koberda, Moses, Koberda, and Koberda (2012a), Rutter (2011), and Wigton (2010). Nevertheless, while it is hoped there are more studies in preparation, currently, there are few examples of empirical investigations of ZNF in peer-reviewed literature evaluating the outcomes of ZNF. There are two such publications that investigate 4ZNF, the first of which is a study by Collura, Guan, Tarrant, Bailey, and Starr (2010) that presented a collection of clinical reports from six clinicians covering 24 successful cases, with an average of 21 sessions per case, and all clients reported clinical improvement. The second publication, by Hammer, Colbert, Brown, and Ilioi (2011), is a randomized, controlled, single-blind study with small sample size $(n=3$ and $n=5)$ that suggests $4 Z N F$ is beneficial for insomnia. For 19ZNF, examples include two single case study evaluations: Hallman (2012) described a case of a child with fetal alcohol syndrome where 80 sessions of 19ZNF brought about remarkable improvements and QEEG normalization; then, Koberda, Moses, Koberda, and Koberda (2012b) described a case where both 19ZNF and LZNF were used with a 23-year-old male, where improvements in cognitive and QEEG assessments were achieved in 15 sessions. For LZNF, one study presented a review of four cases: Koberda, Koberda, Bienkiewicz, Moses, and Koberda (2013) applied LZNF to treat chronic pain and all cases reported improvement.

In terms of number of sessions, ZNF employs simultaneous training of multiple $z$-score metrics (i.e., power, connectivity) at many more electrode sites (up to 19), within either surface or LORETA frameworks. This allows for more neuroregulation and enhanced QEEG normalization. Consequently, in clinical reports of ZNF it is suggested that positive clinical outcomes can be achieved in an average of 10 to 20 sessions (Collura et al., 2010; Koberda et al., 2012b; Wigton, 2013). 
The available $z$-score metrics and multiple channels of the ZNF modalities greatly enhance NF specificity. With surface 4ZNF and 19ZNF, the dysregulated areas and rhythms can be isolated for normalization, and even more so with the Laplacian montage (Thatcher, 2013). With LZNF, brain networks and hubs can be identified and targeted as regions of interest for training. By directly targeting these regions of interest, in a $z$-score framework, it is reported that LZNF allows for specificity and localization similar to that of fMRI methods (Thatcher, 2013). This allows for the linkage of functional systems with the presenting clinical symptoms, such as when identified brain regions (with deviant $z$-scores) are being reinforced towards the mean (i.e., $z=0$ ) to promote increased stability and homeostasis in brain function (Koberda et al., 2013).

\section{Conclusion}

In sum, while all NF is grounded in operant conditioning, it is clear there is a wide range of approaches today. As a result, misunderstandings and misinterpretations can happen if the modality being addressed is not noted. This can have implications for clinical practice, research, and marketing, as has been addressed through this review. For instance, although the AAP recommended that biofeedback/NF be elevated to a Best Support intervention, would all NF modalities be paid if NF is accounted for as a managed care paid intervention (even though the decision seems to be based on traditional NF)? In research, each NF modality may have different levels of efficacy, number of sessions, and treatment specificity, which directly impacts methodology and implications. And, in marketing, a frequent question of NF consumers is how long does the treatment take or how many sessions are needed? In answering these questions, it is clear that the treatment and amount of sessions is guided by the NF modality utilized. Thus, when discussing NF, does modality matter? Yes, it does matter.

\section{References}

American Academy of Pediatrics (AAP). (2012). Evidence-based child and adolescent psychosocial interventions. [chart from: Addressing Mental Health Concerns in Primary Care: A Clinician's Toolkit]. Elk Grove Village, IL: Author.

Arns, M., de Ridder, S., Strehl, U., Breteler, M., \& Coenen. A. (2009). Efficacy of neurofeedback treatment in ADHD: The effects on inattention, impulsivity and hyperactivity: A meta-analysis. Clinical EEG and Neuroscience, 40(3), 180-189. http://dx.doi.org/10.1177/155005940904000311

Arns, M., Drinkenburg, W., \& Kenemans, J. L. (2012). The effects of QEEG-informed neurofeedback in ADHD: An open-label pilot study. Applied Psychophysiology and Biofeedback, 37, 171-180. http://dx.doi.org/10.1007/s10484-012-9191-4

Arns M., Heinrich H., \& Strehl U. (2014). Evaluation of neurofeedback in ADHD: The long and winding road. Biological Psychology, 95, 108-15. http://dx.doi.org/10.1016/j.biopsycho.2013.11.013

Baehr, E., Rosenfeld, J. P., \& Baehr, R. (1997). The clinical use of an alpha asymmetry protocol in the neurofeedback treatment of depression: Two case studies. Journal of Neurotherapy, 2(3), 10-23. http://dx.doi.org/10.1300/J184v02n03 02 


\section{NeuroRegulation}

Beauregard, M., \& Levesque, J. (2006). Functional magnetic resonance imaging investigation of the effects of neurofeedback training on neural bases of selective attention and response inhibition in children with attention-deficit/hyperactivity disorder. Applied Psychophysiology and Biofeedback, 31(1), 3-20. http://dx.doi.org/10.1007/s10484-006-9001-y

Brandeis, D. (2011). Neurofeedback training in ADHD: More news on specificity. Clinical Neurophysiology, 122, 856-857. http://dx.doi.org/10.1016/j.clinph.2010.08.011

Breteler, M. H. M., Arns, M., Peters, S., Giepmans, I., \& Verhoeven, L. (2010). Improvements in spelling after QEEG-based neurofeedback in dyslexia: A randomized controlled treatment study. Applied Psychophysiology and Biofeedback, 35, 5-11. http://dx.doi.org/10.1007/s10484-009-9105-2

Budzynski, T. H. (1999). From EEG to neurofeedback. In J. R. Evans \& A. Abarbanel (Eds.), Introduction to quantitative EEG and neurofeedback (pp. 65-79). San Diego, CA: Academic Press.

Cannon, R., Baldwin, D. R., Diloreto, D. J., Phillips, S. T., Shaw, T. L., \& Levy, J. J. (2014). LORETA neurofeedback in the precuneus: Operant conditioning in basic mechanisms of self-regulation. Clinical EEG and Neuroscience, (Epub ahead of print, March 3, 2014).

Cannon, R., Congedo, M., Lubar, J., \& Hutchens, T. (2009). Differentiating a network of executive attention: LORETA neurofeedback in anterior cingulate and dorsolateral prefrontal cortices. International Journal of Neuroscience, 119(3), 404-441. http://dx.doi.org/10.1080/00207450802480325

Cannon, R., Lubar, J. F., Sokhaze, E., Baldwin, D. (2008). LORETA neurofeedback for addiction and the possible neurophysiology of psychological processes influenced: A case study and region of interest analysis of LORETA neurofeedback in right anterior cingulate cortex. Journal of Neurotherapy, 12(4), 227-241. http://dx.doi.org/10.1080/10874200802501948

Coben, R. \& Myers, T. E. (2010). The relative efficacy of connectivity guided and symptom based EEG biofeedback for autistic disorders. Applied Psychophysiology and Biofeedback, 35, 13-23. http://dx.doi.org/10.1007/s10484-009-9102-5

Collura, T. F. (2008a). Neuronal dynamics in relation to normative electroencephalography assessment and training. Biofeedback, 36(4), 134-139.

http://www.resourcenter.net/images/AAPB/Files/Biofeedback/2008/biof-36-04-134139.pdf

Collura, T. F. (2008b). Whole-head normalization using live Z-scores for connectivity training, Part 1. NeuroConnections Newsletter, April, 12, 15, 18-19. http://www.isnr.net/uploads/NeuroConnections/2008/NCApril08.pdf

Collura, T. F. (2014). Technical foundations of neurofeedback. New York, NY: Routledge.

Collura, T. F., Guan, J. G., Tarrant, J., Bailey, J., \& Starr, F. (2010). EEG biofeedback case studies using live z-score training and a normative database. Journal of Neurotherapy, 14(1), 22-46. http://dx.doi.org/10.1080/10874200903543963

55 | NeuroRegulation

Vol. 1(1):48-60 2014

doi:10.15540/nr.1.1.48

http://www.neuroregulation.org 
Collura, T. F., Thatcher, R. W., Smith, M. L., Lambos, W. A., \& Stark, C. R. (2009). EEG biofeedback training using live z-scores and a normative database. In T. H. Budzynski, H. K. Budzynski, J. R. Evans, \& A. Abarbanel (Eds.), Introduction to quantitative EEG and neurofeedback: Advanced theory and applications (2nd ed., pp. 29-59). Burlington, MA: Elsevier.

Evans, R. J., \& Abarbanel, A. (Eds.). (1999). Introduction to quantitative EEG and neurofeedback. San Diego, CA: Academic Press.

Genardi, D. (2012). BrainAvatar: Flexible individualization for treating Autism, a complex disorder. NeuroConnections, Winter, 13, 15-17, 19, 33. http://www.isnr.net/uploads/NeuroConnections/2012/NCWin12.pdf

Gevensleben, H., Holl, B., Albrecht, B., Vogel, C., Schlamp, D., Kratz, O., ... Heinrich, H. (2009). Is neurofeedback an efficacious treatment for ADHD?: A randomized controlled clinical trial. Journal of Child Psychology and Psychiatry, 50, 780-789. http://dx.doi.org/10.1111/j.1469-7610.2008.02033.x

Gevensleben, H., Rothenberger, A., Moll, G. H., \& Heinrich, H. (2012). Neurofeedback in children with ADHD: Validation and challenges. Expert Reviews of Neurotherapeutics, 12(4), 447-460. http://dx.doi.org/10.1586/ERN.12.22

Hallman, D. W. (2012). 19-Channel neurofeedback in an adolescent with FASD. Journal of Neurotherapy, 16(2), 150-154. http://dx.doi.org/10.1080/10874208.2012.677646

Hammer, B., Colbert, A., Brown, K., \& Ilioi, E. (2011). Neurofeedback for insomnia: A pilot study of z-score SMR and individualized protocols. Applied Psychophysiology and Biofeedback, 36(4), 251-64. http://dx.doi.org/10.1007/s10484-011-9165-y

Hammond, C. D. (2010). The need for individualization in neurofeedback: Heterogeneity in QEEG patterns associated with diagnoses and symptoms. Applied Psychophysiology and Biofeedback, 35(1), 31-36. http://dx.doi.org/10.1007/s10484-009-9106-1

Huang-Storms, L., Bodenhamer-Davis, E., Davis, R., \& Dunn, J. (2006). QEEG-guided neurofeedback for children with histories of abuse and neglect: Neurodevelopmental rationale and pilot study. Journal of Neurotherapy, 10(4), 3-16. http://dx.doi.org/10.1300/J184v10n04 02

Kamiya, J. (1968). Conscious control of brainwaves. Psychology Today, 1, 56-60.

Koberda, J. L. (2012). Autistic spectrum disorder (ASD) as a potential target of z-score LORETA neurofeedback. NeuroConnections, Winter, 24-25. http://www.isnr.net/uploads/NeuroConnections/2012/NCWin12.pdf

Koberda, J. L., Hillier, D. S., Jones, B., Moses, A., \& Koberda, L. (2012). Application of neurofeedback in general neurology practice. Journal of Neurotherapy, 16(3), 231234. http://dx.doi.org/10.1080/10874208.2012.705770 


\section{NeuroRegulation}

Koberda, J. L., Koberda, P., Bienkiewicz, A. A., Moses, A., \& Koberda, L. (2013). Pain management using 19-electrode z-score LORETA neurofeedback. Journal of Neurotherapy, 17(3), 179-190. http://dx.doi.org/10.1080/10874208.2013.813204

Koberda, J. L., Moses, A., Koberda, P., \& Koberda, L. (2012a, September). Comparison of the effectiveness of Z-score surface/LORETA 19-electrodes neurofeedback to standard 1-electrode neurofeedback. Oral Presentation at the $20^{\text {th }}$ Annual Conference of the International Society for Neurofeedback and Research, Orlando, FL.

Koberda, J. L., Moses, A., Koberda, L., \& Koberda, P. (2012b). Cognitive enhancement using 19-electrode z-score neurofeedback. Journal of Neurotherapy, 16(3), 224-230. http://dx.doi.org/10.1080/10874208.2012.705770

Levesque, J., Beauregard, M., \& Mensour, B. (2006). Effect of neurofeedback training on the neural substrates of selective attention in children with attention deficit/hyperactivity disorder: A functional magnetic resonance imaging study. Neuroscience Letters, 394, 216-221. http://dx.doi.org/10.1016/j.neulet.2005.10.100

Lofthouse, N., Arnold, L. E., Hersch, S., Hurt, E., \& DeBeus, R. (2012). A review of neurofeedback treatment for pediatric ADHD. Journal of Attention Disorders, 16, 351-372. http://dx.doi.org/10.1177/1087054711427530

Lubar, J. F., \& Shouse, M. N. (1976). EEG and behavioral changes in a hyperactive child concurrent with training of the sensorimotor rhythm (SMR): A preliminary report. Biofeedback \& Self-Regulation, 1, 293-306. http://dx.doi.org/10.1007/bf01001170

Mayer, K., Wyckoff, S. N., \& Strehl, U. (2013). One size fits all? Slow cortical potentials neurofeedback: A review. Journal of Attention Disorders, 17(5), 393-409. http://dx.doi.org/10.1177/1087054712468053

Niv, S. (2013). Clinical efficacy and potential mechanisms of neurofeedback. Personality and Individual Differences, 54, 676-686. http://dx.doi.org/10.1016/j.paid.2012.11.037

Orgim, G. \& Kestad, K. A. (2013). Effects of neurofeedback versus stimulant medication in attention-deficit/hyperactivity disorder: A randomized pilot study. Journal of Child and Adolescent Psychopharmacology, 23(7), 448-57. http://dx.doi.org/10.1089/cap.2012.0090

Othmer, S., Othmer, S. F., \& Kaiser, D. A. (1999). EEG biofeedback: An emerging model for its global efficacy. In J. R. Evans \& A. Abarbanel (Eds.), Introduction to quantitative EEG and neurofeedback (pp. 243-310). San Diego, CA: Academic Press.

Peniston, E. G., \& Kullkosky, P. J. (1990). Alcoholic personality and alpha-theta brainwave training. Medical Psychotherapy, 3, 37-55.

Peniston, E. G., \& Kullkosky, P. J. (1991). Alpha-theta brainwave neuro-feedback therapy for Vietnam veterans with combat-related post-traumatic-stress disorder. Medical Psychotherapy, 4, 47-60.

Pigott, H. E., De Biase, L., Bodenhamer-Davis, E., \& Davis, R. E. (2013). The evidence-base for neurofeedback as a reimbursable healthcare service to treat attention

57 | NeuroRegulation

Vol. 1(1):48-60 2014

doi:10.15540/nr.1.1.48

http://www.neuroregulation.org 


\section{NeuroRegulation}

deficit/hyperactivity disorder. Retrieved from International Society for Neurofeedback and Research website: http://www.isnr.org/uploads/nfb-adhd.pdf

PracticeWise. (2012, October 01). RE: PracticeWise Fall 2012 Update [electronic mail from Support@PracticeWise.com].

Robbins, J. (2000). A Symphony in the brain. New York, NY: Grove Press.

Roberts, L., Rockstroh. B., Lutzenberger. W., Elbert, T. \& Birbaumer, N. (1989). Self-reports during feedback regulation of slow cortical potentials. Psychophysiology, 26, 392403. http://dx.doi.org/10.1111/j.1469-8986.1989.tb01941.x

Rockstroh, B., Birbaumer, N., Elbert, T., \& Lutzenberger, W. (1984). Operant control of EEG, event-related and slow potentials. Biofeedback and Self-Regulation, 9, 139-160. http://dx.doi.org/10.1007/bf00998830

Rockstroh, B., Elbert, T., Birbaumer, N., Wolf, P., Düchting-Röth, A., Reker, M., \& ... Dichgans, J. (1993). Cortical self-regulation in patients with epilepsies. Epilepsy Research, 14(1), 63-72. http://www.ub.uni-konstanz.de/kops/volltexte/2008/6404/

Rutter, P. (2011, September). Potential clinical applications for 19 channel live z-score training using Percent ZOK and ZPlus protocols. Oral Presentation at the $19^{\text {th }}$ Annual Conference of the International Society for Neurofeedback and Research, Carefree, AZ.

Sherlin, L. H., Arns, M., Lubar, J., Heinrich, H., Kerson, C., Strehl, U., \& Sterman, M. B. (2011). Neurofeedback and basic learning theory: Implications for research and practice. Journal of Neurotherapy, 15(4), 292-304. http://dx.doi.org/10.1080/10874208.2011.623089

Sterman, M. B., \& Friar, L. (1972). Suppression of seizures in an epileptic following sensorimotor EEG feedback training. Electroencephalography and Clinical Neurophysiology, 33(1), 89-95. http://dx.doi.org/10.1016/0013-4694(72)90028-4

Surmeli, T., \& Ertem, A. (2007). EEG neurofeedback treatment of patients with Down syndrome. Journal of Neurotherapy, 11(1), 63-68. http://dx.doi.org/10.1300/J184v11n01 07

Surmeli, T., \& Ertem, A. (2009). QEEG guided neurofeedback therapy in personality disorders: 13 case studies. Clinical EEG and Neuroscience, 40, 5-10. http://dx.doi.org/10.1177/155005940904000107

Surmeli, T., \& Ertem, A. (2010). Post WISC-R and TOVA improvement with QEEG guided neurofeedback training in mentally retarded: A clinical case series of behavioral problems. Clinical EEG and Neuroscience, 41(1), 32-41. http://dx.doi.org/10.1177/155005941004100108

Surmeli, T., \& Ertem, A. (2011). Obsessive compulsive disorder and the efficacy of qEEGguided neurofeedback treatment: A case series. Clinical EEG and Neuroscience, 42(3), 195-201. http://dx.doi.org/10.1177/155005941104200310 


\section{NeuroRegulation}

Surmeli, T., Ertem, A., Eralp, E., \& Kos, I. H. (2012). Schizophrenia and the efficacy of qEEG guided neurofeedback treatment: A clinical case series. Clinical EEG and Neuroscience, 43, 133-144. http://dx.doi.org/10.1177/1550059411429531

Thatcher, R. W. (2008). Z-score EEG biofeedback: Conceptual foundations. NeuroConnections Newsletter , April, 9, 11, 20. San Rafael, CA: International Society for Neurofeedback and Research. http://www.isnr.net/uploads/NeuroConnections/2008/NCApril08.pdf

Thatcher, R. W. (2012). Handbook of quantitative electroencephalography and EEG biofeedback. St. Petersburg, Florida: Anipublishing.

Thatcher, R. W. (2013). Latest developments in live z-score training: Symptom check list, phase reset, and LORETA z-score biofeedback. Journal of Neurotherapy, 17(1), 6987. http://dx.doi.org/10.1080/10874208.2013.759032

Thatcher, R. W., \& Lubar, J. E. (2009). History of the scientific standards of QEEG normative databases. In T. H. Budzynski, H. K. Budzynski, J. R. Evans, \& A. Abarbanel (Eds.), Introduction to quantitative EEG and neurofeedback: Advanced theory and applications (2nd ed., pp. 29-59). Burlington, MA: Elsevier. http://dx.doi.org/10.1016/b978-0-12-374534-7.00002-2

Walker, J. E. (2009). Anxiety associated with post traumatic stress disorder: The role of quantitative electroencephalograph in diagnosis and in guiding neurofeedback training to remediate the anxiety. Biofeedback, 37, 67-70. http://dx.doi.org/10.5298/1081-5937-37.2.67

Walker, J. E. (2010a). Recent advances in quantitative EEG as an aid to diagnosis and as a guide to neurofeedback training for cortical hypofunctions, hyperfunctions, disconnections, and hyperconnections: Improving efficacy in complicated neurological and psychological disorders. Applied Psychophysiology and Biofeedback, 35, 25-27. http://dx.doi.org/10.1007/s10484-009-9107-0

Walker, J. E. (2010b). Using QEEG-guided neurofeedback for epilepsy versus standardized protocols: Enhanced effectiveness? Applied Psychophysiology and Biofeedback, 35, 29-30. http://dx.doi.org/10.1007/s10484-009-9123-0

Walker, J. E. (2011). QEEG-guided neurofeedback for recurrent migraine headaches. Clinical EEG and Neuroscience, 42(1), 59-61. http://dx.doi.org/10.1177/155005941104200112

Walker, J. E. (2012a). Remediation of enuresis using QEEG-guided neurofeedback training. Biofeedback, 40(3), 109-112. http://dx.doi.org/10.5298/1081-5937-40.3.04

Walker, J. E. (2012b). QEEG-guided neurofeedback for remediation of dysgraphia. Biofeedback 40(3), 113-114. http://dx.doi.org/10.5298/1081-5937-40.3.03

Walker, J. E. (2013). QEEG-guided neurofeedback for anger/anger control disorder. Journal of Neurotherapy, 17(1), 88-92. http://dx.doi.org/10.1080/10874208.2012.705767 
Wigton, N. L. (2010, September). Laplacian z-score neurofeedback: A unique option in the realm of multi-channel z-score neurofeedback. Plenary Session Oral Presentation at the $18^{\text {th }}$ Annual Conference of the International Society for Neurofeedback and Research, Denver, CO.

Wigton, N. L. (2013). Clinical perspectives of 19-channel z-score neurofeedback: Benefits and limitations. Journal of Neurotherapy, 17(4), 259-264.

http://dx.doi.org/10.1080/10874208.2013.847142 


\section{The Biofeedback Certification International Alliance}

Isw't it time you were

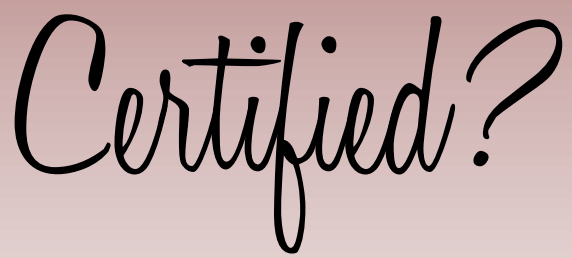

$\mathrm{BCIA}$ is recognized as the gold standard for certification in:

- Biofeedback

- Neurofeedback

- Pelvic Muscle Dysfunction Biofeedback

- BCIA also offers a Certificate in HRV Biofeedback

BCIA also offers a Certificate in HRV Biofeedback

\section{More thaw qualified - BCIA boord certified!}

Mission: $\mathrm{BCIA}$ certifies individuals who meet education and training standards in biofeedback and progressively recertifies those who advance their knowledge through continuing education. 